
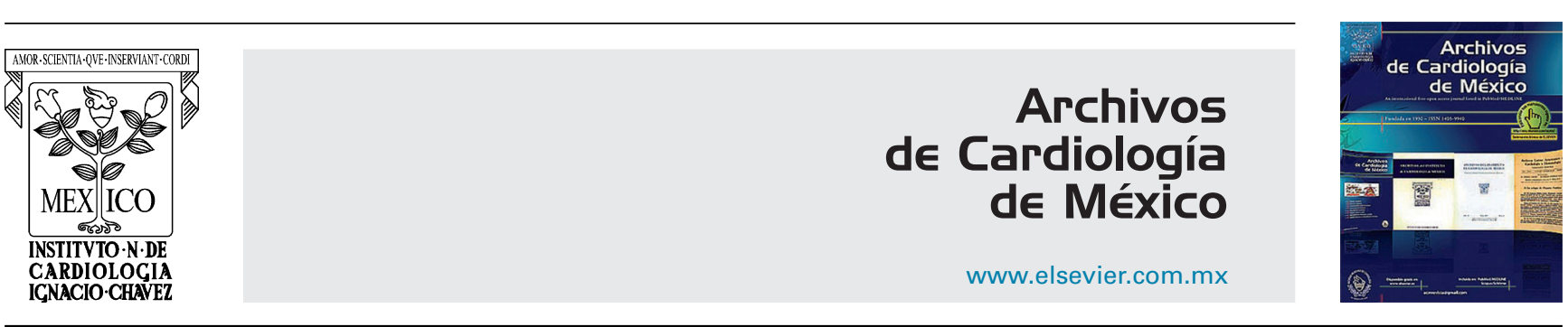

INVESTIGACIÓN CLÍNICA

\title{
Herramientas de sistemas inteligentes en el diagnóstico de los síndromes coronarios agudos: una revisión sistemática
}

\author{
John Sprockel $^{\mathrm{a}, *}$, Miguel Tejeda $^{\mathrm{a}}$, José Yate ${ }^{\mathrm{a}}$, Juan Diaztagle ${ }^{\mathrm{a}, \mathrm{b}}$ y Enrique González \\ a Departamento de Medicina Interna, Hospital de San José, Fundación Universitaria de Ciencias de la Salud, Bogotá, Colombia \\ b Departamento de Ciencias Fisiologicas, Universidad Nacional de Colombia, Bogotá, Colombia \\ c Departamento de Ingeniería de Sistemas, Pontificia Universidad Javeriana, Bogotá, Colombia
}

Recibido el 11 de mayo de 2016; aceptado el 1 de marzo de 2017

\author{
PALABRAS CLAVE \\ Diagnóstico; \\ Infarto de miocardio; \\ Angina inestable; \\ Inteligencia artificial; \\ Colombia
}

\begin{abstract}
Resumen
Antecedentes: El infarto agudo de miocardio representa la primera causa de muerte no trasmisible a nivel mundial. Su diagnóstico es una tarea altamente compleja que se ha intentado modelar mediante métodos automáticos. Se expone una revisión sistemática de estudios de pruebas diagnósticas de los síndromes coronarios agudos mediante sistemas inteligentes.

Métodos: Revisión sistemática de la literatura a partir de Medline, Embase, Scopus, IEEE/IET Electronic Library, ISI Web Of Science, Latindex y LILACS de la evaluación diagnóstica de los síndromes coronarios agudos mediante sistemas inteligentes. Fue realizada por 2 revisores de manera independiente y las discrepancias se resolvieron por una tercera persona. Se extrajeron las características operativas de cada herramienta.

Resultados: En total, 35 artículos cumplieron los criterios de inclusión. En 22 (62.8\%) se utilizaron redes neuronales. Cinco comparan varias herramientas de sistemas inteligentes. En 13 se abarcaba todos los síndromes coronarios agudos y en 22 solo los infartos. En 21 los datos de entrada fueron la clínica y el electrocardiograma, en 10 solo el electrocardiograma. La mayoría utilizan como referente estándar el contexto clínico. Se encontraron altos niveles de precisión diagnóstica con un mejor rendimiento en el caso de redes neuronales y máquinas de soporte de vectores en comparación con las herramientas estadísticas de reconocimiento de patrones y árboles de decisiones.

Conclusiones: Encontramos una amplia evidencia de que los abordajes a través de las herramientas de sistemas inteligentes alcanzan un alto nivel de precisión por lo que deberían ser consideradas como herramientas para el soporte de las decisiones diagnósticas de los síndromes coronarios agudos.

(C) 2017 Instituto Nacional de Cardiología Ignacio Chávez. Publicado por Masson Doyma México S.A. Este es un artículo Open Access bajo la licencia CC BY-NC-ND (http://creativecommons. org/licenses/by-nc-nd/4.0/).
\end{abstract}

\footnotetext{
* Autor para correspondencia. Cra. 19 No. 8. ${ }^{\mathrm{a}}$-32 Pbx: 3538100.

Correo electrónico: jjsprockel@fucsalud.edu.co (J. Sprockel).
} 


\section{KEYWORDS}

Diagnosis;

Myocardial infarction;

Unstable angina;

Artificial intelligence;

Colombia
Intelligent systems tools in the diagnosis of acute coronary syndromes:

A systemic review

\begin{abstract}
Background: Acute myocardial infarction is the leading cause of non-communicable deaths worldwide. Its diagnosis is a highly complex task, for which modelling through automated methods has been attempted. A systematic review of the literature was performed on diagnostic tests that applied intelligent systems tools in the diagnosis of acute coronary syndromes.

Methods: A systematic review of the literature is presented using Medline, Embase, Scopus, IEEE/IET Electronic Library, ISI Web of Science, Latindex and LILACS databases for articles that include the diagnostic evaluation of acute coronary syndromes using intelligent systems. The review process was conducted independently by 2 reviewers, and discrepancies were resolved through the participation of a third person. The operational characteristics of the studied tools were extracted.

Results: A total of 35 references met the inclusion criteria. In $22(62.8 \%)$ cases, neural networks were used. In five studies, the performances of several intelligent systems tools were compared. Thirteen studies sought to perform diagnoses of all acute coronary syndromes, and in 22 , only infarctions were studied. In 21 cases, clinical and electrocardiographic aspects were used as input data, and in 10, only electrocardiographic data were used. Most intelligent systems use the clinical context as a reference standard. High rates of diagnostic accuracy were found with better performance using neural networks and support vector machines, compared with statistical tools of pattern recognition and decision trees.

Conclusions: Extensive evidence was found that shows that using intelligent systems tools achieves a greater degree of accuracy than some clinical algorithms or scales and, thus, should be considered appropriate tools for supporting diagnostic decisions of acute coronary syndromes. (c) 2017 Instituto Nacional de Cardiología Ignacio Chávez. Published by Masson Doyma México S.A. This is an open access article under the CC BY-NC-ND license (http://creativecommons. org/licenses/by-nc-nd/4.0/).
\end{abstract}

\section{Introducción}

La enfermedad cardiovascular es la principal causa de muerte en los países industrializados y se espera que para el año 2020 también lo sea para los países en vía de desarrollo ${ }^{1}$. Una de sus formas de presentación, los síndromes coronarios agudos (SCA), es la principal causa mortalidad. Este síndrome es una entidad con un alto grado de complejidad que para su diagnóstico requiere considerar los datos de la historia clínica, los antecedentes personales y familiares, en conjunto con varias ayudas diagnósticas sustentadas principalmente en el electrocardiograma (ECG), biomarcadores, las pruebas de estratificación no invasivas y la angiografía coronaria. Por lo general, debe llevarse a cabo en el ambiente de urgencias en el que la rapidez del diagnóstico es esencial y existe además una amplia variedad de diagnósticos diferenciales que incluyen otras condiciones que pueden poner en peligro la vida del paciente.

Los esfuerzos en la atención clínica incluyen de manera cada vez más frecuente el uso de instrumentos de gestión electrónica, dentro de las que se incluyen las herramientas inteligentes, con el objetivo de mejorar los tiempos y la precisión en el diagnóstico para brindar una servicio de mayor calidad constituyendo esto un paradigma en el modelo de atención que incluye un soporte a las decisiones clínicas ${ }^{2}$. Se entiende como «sistemas inteligentes» aquellos intentos de emular la inteligencia humana, los cuales favorecen el razonamiento y aprendizaje en ambientes inciertos o imprecisos ${ }^{3}$, término que es sinónimo, hasta cierto punto, de los de inteligencia artificial, inteligencia computacional y reconocimiento de patrones. Está constituido principalmente por: sistemas basados en reglas (o sistemas expertos), redes neuronales artificiales (ANN), computación evolutiva, lógica difusa, máquinas de soporte de vectores (SVM), redes de creencia bayesianas, árboles de decisiones, sistemas de ensamble como Ada-Boost y Bagging, sistemas multiagentes (o agentes racionales colaborativos) entre otros.

Una revisión sistemática llevada a cabo en el 2010, en la que se evaluó el valor de los instrumentos para descartar los SCA, encontró que ninguna de ellas logró cumplir con los requerimientos de seguridad para los clínicos al momento de soportar el diagnóstico ${ }^{4}$. Dicha revisión incluyó solo herramientas basadas en reglas diseñadas por los médicos y modelos de regresión logística; no se tuvo en cuenta las que estaban basadas en sistemas inteligentes. Ninguna de las guías de práctica clínica actual que abordan el diagnóstico del dolor torácico hace alguna consideración acerca de su uso ${ }^{5-7}$.

Al tratarse de un problema altamente complejo, el diagnóstico médico ha generado la necesidad de buscar una forma para lograr su estandarización como proceso, se ha convertido en un campo de estudio para la aplicación de los sistemas inteligentes, por lo que su aplicación reviste interés especial en la entidad clínica responsable de la mayor cantidad de muertes en el mundo. Es por ello que en el presente trabajo se lleva a cabo una revisión sistemática de la 
literatura de los estudios de pruebas diagnósticas que evalúen la aplicación de herramientas de sistemas inteligentes en el diagnóstico de los SCA, las cuales puedan ser aplicadas al paciente que consulta por dolor torácico a urgencias.

\section{Metodología}

\section{Estrategia de búsqueda}

Se consultaron las siguientes bases de datos; Medline, Embase, Scopus, IEEE/IET Electronic Library e ISI Web Of Science, Latindex y LILACS; la búsqueda incluyó información hasta abril de 2014 y se limitó a estudios publicados en inglés o en español. En el material suplementario se exponen los términos usados en cada base de datos. La condición a estudio fueron los SCA que se encuentran constituidos por el infarto agudo de miocardio con y sin elevación del segmento ST así como por la angina inestable. Se tomó como prueba índice los diferentes aplicativos con el uso de sistemas inteligentes para el apoyo diagnóstico de los SCA.

Al poner en consideración la evolución temporal del diagnóstico de los SCA, principalmente del infarto, se decidió no limitar el referente de diagnóstico a un conjunto predefinido de estudios revisando que fueran apropiados para la época. En todo caso se describen rigurosamente dentro de los resultados los criterios de cada estudio para la definición de los casos.

\section{Criterios de inclusión}

Se incluyeron todos los estudios publicados que utilizaron herramientas de sistemas inteligentes para el diagnóstico de los SCA independientemente de la duración del seguimiento o el estatus de la publicación. Solo se incluyeron los artículos en idioma inglés o español.

\section{Criterios de exclusión}

No se consideraron aquellos estudios que evaluaron: solo el ECG mediante procesamiento directo de la señal, estudios de Holter, formas alternas para su toma (p. ej.: ECG ortogonal, magnetocardiogramas) o procesamiento alterno de la señal de este (p. ej.: transformación discreta de ondas), el resultados de la prueba de esfuerzo convencional (ECG de ejercicio), reconocimiento de imágenes de perfusiones miocárdicas, tomografía, resonancia magnética nuclear o angiografía coronaria invasiva, telemetría u otros conceptos de computación ubicua, evaluaciones de factores de riesgo o pronóstico del SCA o intervenciones terapéuticas, se descartaron los que evaluaban el síndrome de muerte súbita, debido a que corresponde a un espectro diferente de los SCA.

\section{Selección de los estudios y extracción de los datos}

Se hizo una selección de los artículos que cumplieran los criterios de inclusión para esta revisión mediante la lectura del título y el resumen; para los artículos restantes se procedió a la lectura del texto completo. Este proceso fue ejecutado por 2 revisores de manera independiente (MT y AY). Las discrepancias se resolvieron con la participación de una tercera persona (JS). La extracción de los datos fue llevada a cabo por uno de los revisores (JS) mediante un formato establecido previamente que reunió las características de la población, de la herramienta utilizada y la exactitud diagnóstica. Se extrajeron las características operativas de las herramientas estudiadas en términos de sensibilidad, especificidad, valores predictivos o área bajo la curva ROC (receiver-operating characteristic).

\section{Riesgo de sesgos}

Se utilizó la lista de chequeo del Quality Assessment of Diagnostic Accuracy Studies (QUADAS) $2^{8}$ para el cual se realizó una definición operativa de cada uno de los ítems que la constituye.

El estudio fue aprobado por el comité de investigaciones y ética de la Fundación Universitaria de Ciencias de la Salud y el Hospital de San José de Bogotá.

\section{Resultados}

Se identificaron 2,187 referencias de la búsqueda en bases de datos. A partir de los títulos y los resúmenes se seleccionaron 91 para revisión del texto completo, de los cuales 35 artículos cumplieron los criterios de inclusión. En la figura 1 se muestra el flujograma de los artículos que fueron tenidos en cuenta durante el proceso de búsqueda (). La primera tabla resume los diseños empleados y la población sobre la que se desarrolló (tabla 1) y en la segunda se muestran los resultados de su desempeño diagnóstico organizando los artículos en estricto orden cronológico (tabla 2).

Al realizar el análisis del riesgo de sesgos mediante la herramienta QUADAS 2 (tabla 3), podemos notar que en general se obtuvo un bajo riesgo en la mayor parte de los estudios. El problema más frecuentemente descrito se centró en la descripción de la base de datos usada con lo que no quedan claro algunos aspectos de la selección de los pacientes y del referente para el diagnóstico ${ }^{11,14,22-24,37,39-41,43}$ (fig. 2). Los casos de riesgo alto incluyen estudios donde se usó solo las derivación DIII ${ }^{37}$ y V1 a V4 ${ }^{40}$ del ECG para el diagnóstico de los infartos, limitando la posibilidad diagnóstica a un subgrupo de infartos (de pared inferior o anterior respectivamente).

Se encuentra que en 22 (62.8\%) de los casos se utilizaron las redes neuronales como la herramienta de sistemas inteligentes (fig. 3), las cuales fueron evaluadas con experimentos amplios que incluían diversas distribuciones en su arquitectura (números de datos de entrada, capas ocultas, neuronas de salida), algoritmos de entrenamiento, tipo de red (perceptron simple, múltiple o red probabilística ${ }^{29}$ ) y contextos clínicos. En 5 trabajos se comparan los rendimientos de varias herramientas de sistemas inteligentes (uno comparó 8 estrategias) $)^{23,36,40,42,43}$. En los últimos años fueron publicados con mayor frecuencia estudios que evaluaron $\mathrm{SVM}^{31,36,40,41}$ y sistemas de ensamble $\mathrm{e}^{28,34,35}$. En 7 casos se utilizaron estrategias de reconocimiento estadístico de patrones $^{12,22,23,36,40,42,43}$ y un estudió evaluó un sistema de clustering ${ }^{39}$. 


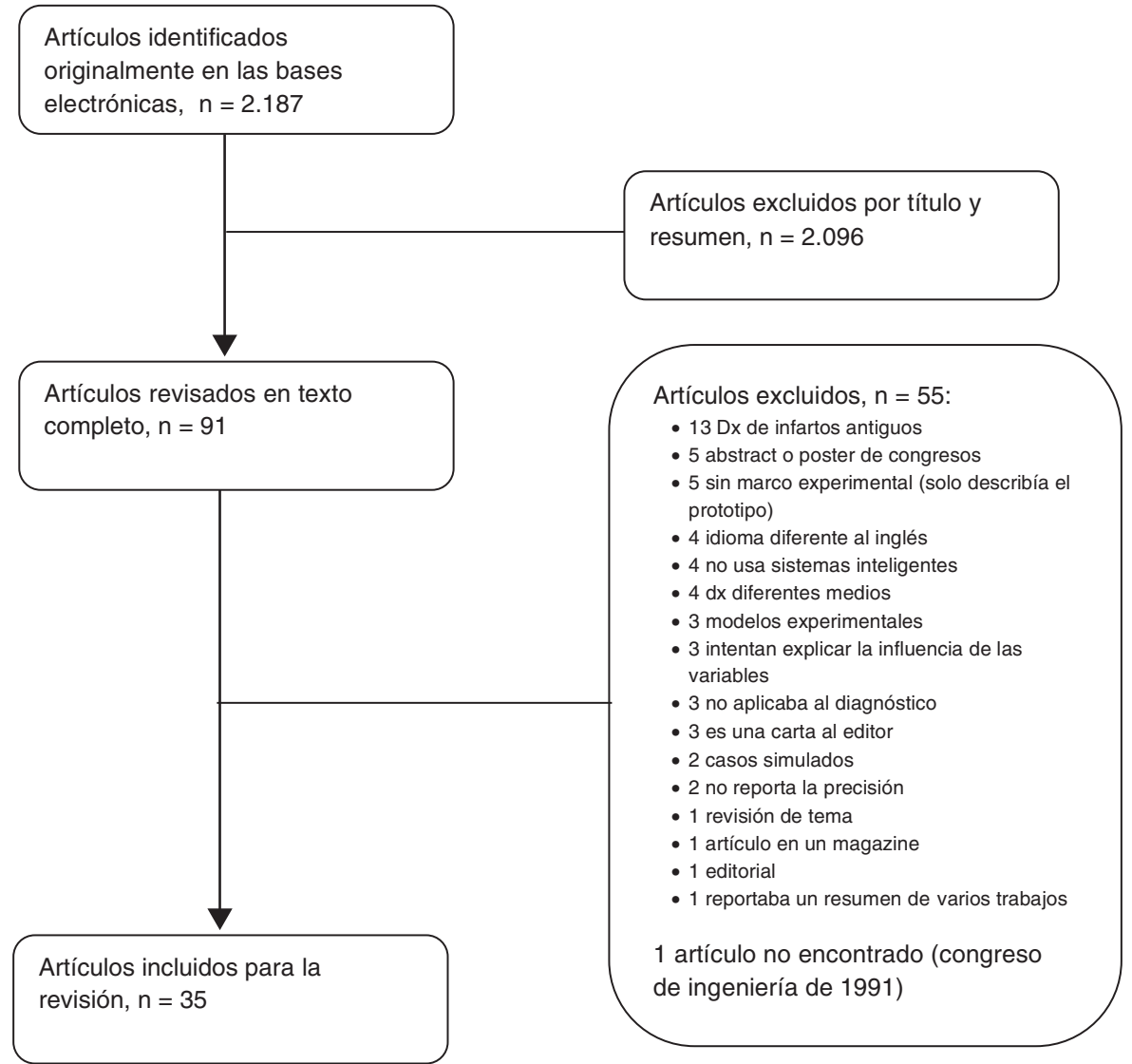

Figura 1 Flujograma de los trabajos durante el proceso de búsqueda.

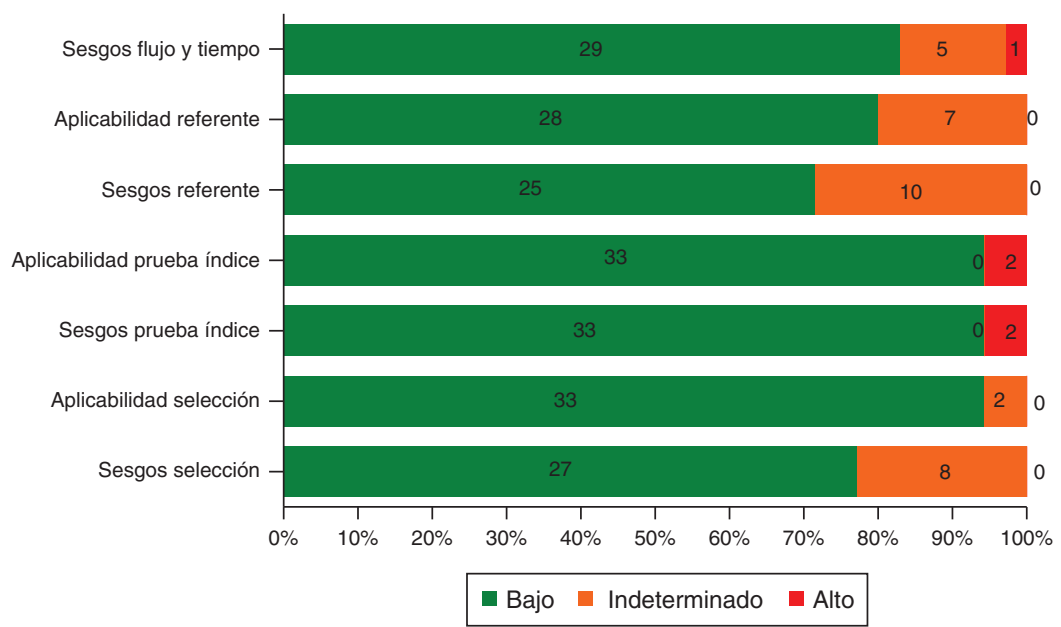

Figura 2 Distribución de las frecuencias acumuladas de los riesgos de sesgos y de aplicabilidad de acuerdo a la herramienta QUADAS 2.

En 13 trabajos se buscaba realizar el diagnóstico de todos los SCA $9,12,13,17,19,22,26,27,30,33,35,38,42$ y en 22 solo de los infartos (uno de ellos en casos de bloqueos completos de rama izquierda ${ }^{28}$ ). En 21 casos se tuvo en cuenta como datos de entrada aspectos clínicos y electrocardiográficos, en 10 solo datos electrocardiográficos ${ }^{14,20,24,28-30,34,38,40,41}$, en 2 solo datos clínicos ${ }^{22,25}$ y en 2 casos solo de biomarcadores de lesión miocárdica ${ }^{11,18}$. La mayoría de los sistemas inteligentes utilizan como referente estándar el contexto clínico dado por la combinación de aspectos clínicos, ECG, biomarcadores junto a alguna estrategia de estratificación; en un estudio estuvo determinado por el concepto de los $\operatorname{cardiólogos}^{18}$, en otro por angiografía coronaria ${ }^{41}$ y en uno se tuvo en cuenta los hallazgos de enfermedad en autopsias ${ }^{11}$. Se desconoce el referente estándar en 4 estudios $^{23,24,31,40}$. En 7 casos se compararon los resultados 
Tabla 1 Estructura y características del entrenamiento de los diferentes sistemas inteligentes

\begin{tabular}{|c|c|c|c|c|c|}
\hline Estudio (ref.) & $\begin{array}{l}\text { Descripción de las herramientas } \\
\text { usadas }\end{array}$ & $\begin{array}{l}\text { Datos de } \\
\text { entrada }\end{array}$ & $\begin{array}{l}\text { Condición } \\
\text { clínica }\end{array}$ & $\begin{array}{l}\text { Referente } \\
\text { estándar }^{\mathrm{a}}\end{array}$ & $\begin{array}{l}\text { Número de } \\
\text { pacientes }^{b}\end{array}$ \\
\hline Baxt, $1991^{c} 9$ & $\begin{array}{l}\text { RN con } 20 \text { entradas, } 2 \text { capas } \\
\text { ocultas con } 10 \text { neuronas cada } \\
\text { una y una salida }\end{array}$ & Clínicos y ECG & Infarto agudo & $\begin{array}{l}\text { Contexto } \\
\text { clínico }\end{array}$ & $\begin{array}{l}351 \text { casos } \\
(50: 50)\end{array}$ \\
\hline $\begin{array}{l}\text { Harrison et al., } \\
\qquad 1991^{\text {d } 10}\end{array}$ & $\begin{array}{l}\text { RN con } 53 \text { entradas, una capa } \\
\text { oculta con } 18 \text { neuronas y una } \\
\text { salida }\end{array}$ & Clínicos y ECG & Infarto agudo & $\begin{array}{l}\text { Contexto } \\
\text { clínico }\end{array}$ & $\begin{array}{l}300 \text { casos } \\
(50: 50)\end{array}$ \\
\hline $\begin{array}{l}\text { Furlong et al., } \\
\qquad 1991^{\text {c }} 11\end{array}$ & $\begin{array}{l}\text { RN con } 21 \text { entradas, una capa } \\
\text { oculta con } 11 \text { neuronas y una } \\
\text { salida }\end{array}$ & Biomarcadores & Infarto agudo & $\begin{array}{l}\text { Contexto } \\
\text { clínico }\end{array}$ & 47 casos \\
\hline$\underset{12}{\text { Aase et al., } 1993^{c}}$ & $\begin{array}{l}\text { Bayes nativo (naïve) a partir de } \\
31 \text { variables }\end{array}$ & Clínicos y ECG & Todos los SCA & $\begin{array}{l}\text { Contexto } \\
\text { clínico }\end{array}$ & 918 pacientes \\
\hline $\begin{array}{l}\text { Baxt y Skora, } \\
1996^{c} 13\end{array}$ & $\begin{array}{l}\text { RN con } 20 \text { entradas, } 2 \text { capas } \\
\text { ocultas con } 10 \text { neuronas cada } \\
\text { una y una salida }\end{array}$ & Clínicos y ECG & Todos los SCA & $\begin{array}{l}\text { Contexto } \\
\text { clínico }\end{array}$ & 1,070 \\
\hline $\begin{array}{l}\text { Bozzola et al., } \\
1996^{\text {d } 14}\end{array}$ & $\begin{array}{l}\text { Sistema híbrido neurofuzzy con } 8 \\
\text { neuronas de entrada }\end{array}$ & ECG & Infarto agudo & $\begin{array}{l}\text { Contexto } \\
\text { clínico }\end{array}$ & $\begin{array}{l}539 \text { casos } \\
(75: 25)\end{array}$ \\
\hline $\begin{array}{l}\text { Jørgensen et al., } \\
\qquad 1996^{c} 15\end{array}$ & $\begin{array}{l}\text { RN con } 5,10 \text { y } 20 \text { entradas, una } \\
\text { capa oculta y una neurona de } \\
\text { salida }\end{array}$ & Clínicos y ECG & Infarto agudo & $\begin{array}{l}\text { Contexto } \\
\text { clínico }\end{array}$ & $\begin{array}{l}250 \text { casos } \\
(40: 60)\end{array}$ \\
\hline $\begin{array}{l}\text { Pedersen et al., } \\
1996^{c} 16\end{array}$ & $\begin{array}{l}\text { RN con } 5,10 \text { y } 20 \text { entradas, una } \\
\text { capa oculta y una neurona de } \\
\text { salida }\end{array}$ & Clínicos y ECG & Infarto agudo & $\begin{array}{l}\text { Contexto } \\
\text { clínico }\end{array}$ & $\begin{array}{l}250 \text { casos } \\
(40: 60)\end{array}$ \\
\hline $\begin{array}{l}\text { Hirshberg y } \\
\text { Guttman, } 1996^{c} \\
17\end{array}$ & Sistema basado en reglas & Clínicos y ECG & Todos los SCA & $\begin{array}{l}\text { Contexto } \\
\text { clínico }\end{array}$ & 59 casos \\
\hline $\begin{array}{l}\text { Ellenius et al., } \\
\qquad 1997^{\text {d } 18}\end{array}$ & $\begin{array}{l}\text { Varios modelos de RN: } \\
\text { perceptron simple y multicapa }\end{array}$ & Biomarcadores & Infarto agudo & Cardiólogos & $\begin{array}{l}80 \text { casos } \\
(84: 16)\end{array}$ \\
\hline $\begin{array}{l}\text { Kennedy et al., } \\
1997^{\text {d } 19}\end{array}$ & $\begin{array}{l}\text { RN con } 39 \text { entradas, una capa } \\
\text { oculta con } 18 \text { neuronas y una } \\
\text { salida }\end{array}$ & Clínicos y ECG & Todos los SCA & $\begin{array}{l}\text { Contexto } \\
\text { clínico }\end{array}$ & $\begin{array}{l}290 \text { entren, } \\
91 \text { validación }\end{array}$ \\
\hline $\begin{array}{l}\text { Hedén et al., } \\
1997^{c} 20\end{array}$ & $\begin{array}{l}\text { RN con } 72 \text { entradas, una capa } \\
\text { oculta con } 15 \text { neuronas y una } \\
\text { salida }\end{array}$ & ECG & Infarto agudo & $\begin{array}{l}\text { Contexto } \\
\text { clínico }\end{array}$ & 11572 casos \\
\hline$\underset{21}{\text { Tsien et al., } 1998^{c}}$ & $\begin{array}{l}\text { Árbol de decisiones } \mathrm{C} 4,5 \text { con } 45 \\
\text { datos de entrada }\end{array}$ & Clínicos y ECG & Infarto agudo & $\begin{array}{l}\text { Contexto } \\
\text { clínico }\end{array}$ & $\begin{array}{l}1,252 \text { entren, } \\
500 \\
\text { validación }\end{array}$ \\
\hline Aase, $1999^{c} 22$ & $\begin{array}{l}\text { Bayes nativo (naïve) a partir de } \\
38 \text { datos de la historia clínica }\end{array}$ & Clínicos & Todos los SCA & $\begin{array}{l}\text { Contexto } \\
\text { clínico }\end{array}$ & $\begin{array}{l}918 \text { entren y } \\
493 \text { prueba }\end{array}$ \\
\hline $\begin{array}{l}\text { Dreiseitl et al., } \\
\text { 1999с } 23\end{array}$ & $\begin{array}{l}\text { Varios modelos: RN, red } \\
\text { bayesiana y conjuntos de } \\
\text { aproximación (Rough Set). Con } 8 \\
\text { a } 40 \text { datos }\end{array}$ & Clínicos y ECG & Infarto agudo & $\begin{array}{l}\text { No se } \\
\text { describe }\end{array}$ & $\begin{array}{l}500 \text { casos } \\
(67: 33)\end{array}$ \\
\hline Lu et al., $2000^{d} 24$ & $\begin{array}{l}\text { Sistema híbrido neurofuzzy con } \\
22 \text { neuronas de entrada y } 4 \text { capas }\end{array}$ & ECG & Infarto agudo & $\begin{array}{l}\text { No se } \\
\text { describe }\end{array}$ & 124 casos \\
\hline 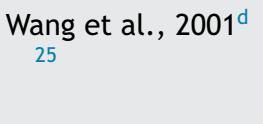 & $\begin{array}{l}\text { RN con } 30 \text { entradas, una capa } \\
\text { oculta con } 15 \text { neuronas y una } \\
\text { salida }\end{array}$ & Clínicos & Infarto agudo & $\begin{array}{l}\text { Contexto } \\
\text { clínico }\end{array}$ & $\begin{array}{l}1,253 \text { entren, } \\
500 \\
\text { validación }\end{array}$ \\
\hline $\begin{array}{l}\text { Baxt et al., } \\
2002-1^{\text {c } 26}\end{array}$ & $\begin{array}{l}\text { RN con } 40 \text { entradas, una capa } \\
\text { oculta con } 10 \text { neuronas y una } \\
\text { salida }\end{array}$ & Clínicos y ECG & Todos los SCA & $\begin{array}{l}\text { Contexto } \\
\text { clínico }\end{array}$ & $2,204(83: 17)$ \\
\hline $\begin{array}{l}\text { Baxt et al., } \\
2002-2^{\text {c } 27}\end{array}$ & $\begin{array}{l}\text { RN con } 40 \text { entradas, una capa } \\
\text { oculta con } 10 \text { neuronas y una } \\
\text { salida }\end{array}$ & Clínicos y ECG & Todos los SCA & $\begin{array}{l}\text { Contexto } \\
\text { clínico }\end{array}$ & $2,204(83: 17)$ \\
\hline
\end{tabular}


Tabla 1 (continuación)

\begin{tabular}{|c|c|c|c|c|c|}
\hline Estudio (ref.) & $\begin{array}{l}\text { Descripción de las herramientas } \\
\text { usadas }\end{array}$ & $\begin{array}{l}\text { Datos de } \\
\text { entrada }\end{array}$ & $\begin{array}{l}\text { Condición } \\
\text { clínica }\end{array}$ & $\begin{array}{l}\text { Referente } \\
\text { estándar }\end{array}$ & $\begin{array}{l}\text { Número de } \\
\text { pacientes }^{\mathrm{b}}\end{array}$ \\
\hline $\begin{array}{l}\text { Olsson et al., } \\
2002^{\text {c } 29}\end{array}$ & $\begin{array}{l}\text { Sistema de ensamble de } 50 \text { RN } \\
\text { cuya arquitectura consistía en } 30 \\
\text { entradas, una capa oculta con } 12 \\
\text { neuronas y una salida }\end{array}$ & ECG & Infarto agudo & $\begin{array}{l}\text { Contexto } \\
\text { clínico }\end{array}$ & $\begin{array}{l}518 \text { casos } \\
(33: 67)\end{array}$ \\
\hline $\begin{array}{l}\text { Haraldsson et al., } \\
2004^{\text {c } 29}\end{array}$ & $\begin{array}{l}\text { RN probabilísticas con } 48 \text { a } 108 \\
\text { entradas, una capa oculta con } 8 \\
\text { neuronas y una salida }\end{array}$ & ECG & Infarto agudo & $\begin{array}{l}\text { Contexto } \\
\text { clínico }\end{array}$ & $\begin{array}{l}2,248 \text { casos } \\
(67: 33)\end{array}$ \\
\hline $\begin{array}{l}\text { Harrison y } \\
\text { Kennedy, 2004c } \\
30\end{array}$ & $\begin{array}{l}\text { RN con } 13 \text { entradas, una capa } \\
\text { oculta con } 10 \text { neuronas y una } \\
\text { salida }\end{array}$ & ECG & Todos los SCA & $\begin{array}{l}\text { Contexto } \\
\text { clínico }\end{array}$ & $\begin{array}{l}1,253 \text { entren, } \\
1,268 \\
\text { validación }\end{array}$ \\
\hline $\begin{array}{l}\text { Conforti y Guido, } \\
2005^{\text {d } 31}\end{array}$ & $\begin{array}{l}\text { SVM con varios tipos de kernel } \\
\text { con } 125 \text { o con } 25 \text { datos de } \\
\text { entrada }\end{array}$ & Clínicos y ECG & Infarto agudo & $\begin{array}{l}\text { No se } \\
\text { describe }\end{array}$ & 242 casos \\
\hline Bulgiba, 2006c 32 & $\begin{array}{l}\text { RN con } 9 \text { datos aportados por el } \\
\text { paciente para tamizaje }\end{array}$ & Clínicos y ECG & Infarto agudo & $\begin{array}{l}\text { Contexto } \\
\text { clínico }\end{array}$ & $710(90: 10)$ \\
\hline $\begin{array}{l}\text { Coskun et al., } \\
2006^{c} 33\end{array}$ & $\begin{array}{l}\text { Árbol de decisiones con } 6 \\
\text { variables, uso de telemedicina }\end{array}$ & Clínicos y ECG & Todos los SCA & $\begin{array}{l}\text { Contexto } \\
\text { clínico }\end{array}$ & $365(68: 32)$ \\
\hline $\begin{array}{l}\text { Olsson et al., } \\
2006^{\text {c } 34}\end{array}$ & $\begin{array}{l}\text { Sistema de ensamble de } 50 \text { RN } \\
\text { con } 27 \text { entradas, una capa oculta } \\
\text { con } 15 \text { neuronas y una salida }\end{array}$ & ECG & Infarto agudo & $\begin{array}{l}\text { Contexto } \\
\text { clínico }\end{array}$ & $\begin{array}{l}4,000 \text { casos } \\
(75: 25)\end{array}$ \\
\hline $\begin{array}{l}\text { Green et al., } \\
2006^{c} 35\end{array}$ & $\begin{array}{l}\text { Ensamble de RN por Bagging y } \\
\text { cross-spliting S-fold. } 38,22 \text { o } 16 \\
\text { variables de entrada }\end{array}$ & Clínicos y ECG & Todos los SCA & $\begin{array}{l}\text { Contexto } \\
\text { clínico }\end{array}$ & 634 casos \\
\hline $\begin{array}{l}\text { Conforti et al., } \\
2007^{c} 36\end{array}$ & $\begin{array}{l}\text { Varios modelos: SVM con varios } \\
\text { kernel, Bayes naïve, árbol de } \\
\text { decisiones y RN }\end{array}$ & Clínicos y ECG & Infarto agudo & $\begin{array}{l}\text { Contexto } \\
\text { clínico }\end{array}$ & 242 casos \\
\hline $\begin{array}{l}\text { Al-Naima et al., } \\
\qquad 2008^{d} 37\end{array}$ & $\begin{array}{l}\text { RN entradas: } 16,21 \text { o } 32,2 \\
\text { capas ocultas con } 5 \text { y } 3 \\
\text { neuronas, una neurona de salida }\end{array}$ & Clínicos y ECG & Infarto agudo & $\begin{array}{l}\text { Contexto } \\
\text { clínico }\end{array}$ & $42(64: 36)$ \\
\hline $\begin{array}{l}\text { Forberg et al., } \\
\qquad 2009^{c} 38\end{array}$ & $\begin{array}{l}\text { RN con } 54 \text { datos } \\
\text { electrocardiográficos de } \\
\text { entrada, una capa oculta con } 10 \\
\text { neuronas. Se comparó con RL }\end{array}$ & ECG & Todos los SCA & $\begin{array}{l}\text { Contexto } \\
\text { clínico }\end{array}$ & 861 casos \\
\hline$\underset{39}{\text { Ainon et al., } 2012^{c}}$ & $\begin{array}{l}\text { Fuzzy C-means Clustering con } \\
48,5 \text { o } 7 \text { datos de entrada }\end{array}$ & Clínicos y ECG & Infarto agudo & $\begin{array}{l}\text { Contexto } \\
\text { clínico }\end{array}$ & 887 casos \\
\hline $\begin{array}{l}\text { Chang et al., } \\
2012^{\mathrm{d}} 40\end{array}$ & $\begin{array}{l}\text { SVM y modelos de mezclas de } \\
\text { gaussianas }\end{array}$ & ECG & Infarto agudo & $\begin{array}{l}\text { No se } \\
\text { describe }\end{array}$ & $\begin{array}{l}1,129 \text { entren } \\
\text { y } 200 \text { pruebas }\end{array}$ \\
\hline $\begin{array}{l}\text { Dhawan et al., } \\
\qquad 2012^{\mathrm{d}} 41\end{array}$ & $\begin{array}{l}\text { SVM multicapa con kernel de } \\
\text { base radial en } 4 \text { capas }\end{array}$ & ECG & Infarto agudo & Cateterísmo & 201 casos \\
\hline$\underset{42}{\text { Salari et al., } 2013^{c}}$ & $\begin{array}{l}\text { Ocho estrategias: Modelo } \\
\text { generalizado lineal, k-NN, ANFIS, } \\
\text { Bayes, árbol de decisiones (ID3), } \\
\text { Bagging, RBF y RN }\end{array}$ & Clínicos y ECG & Todos los SCA & $\begin{array}{l}\text { Contexto } \\
\text { clínico más } \\
\text { seguimiento }\end{array}$ & 809 pacientes \\
\hline $\begin{array}{l}\text { Vila-Francés } \\
\text { et al., } 2013^{\text {c } 43}\end{array}$ & $\begin{array}{l}\text { Compara varias estrategias de } \\
\text { reconocimiento estadístico de } \\
\text { patrones: Bayes, FAN, TAN y } \\
\text { redes bayesianas }\end{array}$ & Clínicos y ECG & Infarto agudo & $\begin{array}{l}\text { Contexto } \\
\text { clínico }\end{array}$ & 1,267 casos \\
\hline
\end{tabular}

ANFIS: adaptive neuro fuzzy inference system; ECG: electrocardiograma; FAN: Forest Augmented Network; k-NN: k vecinos cercanos; RBF: función de base radial; RL: regresión logística; RN: red neuronal; SCA: síndromes coronarios agudos; SVM: máquina de soporte de vectores; TAN: Tree Augmented Network.

${ }^{a}$ El contexto clínico hace referencia a la combinación de los datos clínicos, del ECG, biomarcadores y alguna estratificación.

${ }^{b}$ Entre paréntesis se describe cuánto fue el porcentaje tenido en cuenta para el entrenamiento y las pruebas.

c Revistas médicas.

d Revistas de ingeniería. 
Tabla 2 Rendimiento diagnóstico de los sistemas inteligentes

\begin{tabular}{|c|c|c|c|c|c|}
\hline Estudio & Sensibilidad & Especificidad & Precisión & Otro reporte & Comentarios \\
\hline Baxt, 1991 a 9 & 97.2 & 96.2 & 96.6 & - & $\begin{array}{l}\text { Fue comparada contra el rendimiento de } \\
\text { los médicos de urgencias }\end{array}$ \\
\hline $\begin{array}{l}\text { Harrison et al., } \\
1991^{\text {b } 10}\end{array}$ & 96 & 86 & 89 & - & $\begin{array}{l}\text { Con los datos clínicos se mejora el } \\
\text { desempeño }\end{array}$ \\
\hline $\begin{array}{l}\text { Furlong et al., } \\
\qquad 1991^{\text {a }} 11\end{array}$ & 85.7 & 66.7 & 82.3 & - & $\begin{array}{l}\text { Entrenó RN con biomarcadores seriados } \\
\text { (CPK y LDH). Analizó una subpoblación } \\
\text { en que se realizó un diagnóstico a partir } \\
\text { de autopsias }\end{array}$ \\
\hline $\begin{array}{l}\text { Aase et al., } \\
1993^{\mathrm{a}} 12\end{array}$ & NR & NR & 89 & - & $\begin{array}{l}\text { Evalúa la influencia del número de } \\
\text { grupos para la clasificación }\end{array}$ \\
\hline $\begin{array}{l}\text { Baxt y Skora, } \\
1996^{\mathrm{a}} 13\end{array}$ & 96 & 96 & 96 & - & Es la validación de un anterior trabajo \\
\hline $\begin{array}{l}\text { Bozzola et al., } \\
\qquad 1996^{\text {b } 14}\end{array}$ & 88 & 94.5 & NR & - & $\begin{array}{l}\text { No se describe claramente cómo se } \\
\text { diagnosticó el infarto ni la red neuronal } \\
\text { con la que se comparó }\end{array}$ \\
\hline $\begin{array}{l}\text { Jørgensen et al., } \\
\qquad 1996^{\mathrm{a}} 15\end{array}$ & NR & NR & 78 & - & $\begin{array}{l}\text { Hacen una magnífica exposición del } \\
\text { cómo afectan los cambios en la } \\
\text { arquitectura de la RN a su rendimiento } \\
\text { con el ejemplo de los infartos }\end{array}$ \\
\hline $\begin{array}{l}\text { Pedersen et al., } \\
1996^{a} 16\end{array}$ & $\begin{array}{l}\text { Ingreso: } 79 \% \\
24 \mathrm{~h}: 99 \%\end{array}$ & $\begin{array}{l}\text { Ingreso: } 84 \% \\
24 \mathrm{~h}: 99 \%\end{array}$ & $\begin{array}{l}\text { Ingreso: } \\
81 \% 24 \mathrm{~h}: \\
99 \%\end{array}$ & - & $\begin{array}{l}\text { Hace un análisis con los datos al ingreso, } \\
\text { a las } 12 \text { y } 24 \mathrm{~h} \text {. Comparado con análisis } \\
\text { discriminante que fue ligeramente } \\
\text { inferior }\end{array}$ \\
\hline $\begin{array}{l}\text { Hirshberg y } \\
\text { Guttman, } \\
1996^{\text {a } 17}\end{array}$ & 100 & 39 & NR & - & $\begin{array}{l}\text { Asigna a los cardiólogos una sensibilidad } \\
\text { del } 100 \% \text { y una especificidad del } 16 \% \text {. } \\
\text { Sistema comercial }\end{array}$ \\
\hline $\begin{array}{l}\text { Ellenius et al., } \\
\qquad 1997^{\mathrm{b}} 18\end{array}$ & 100 & 93 & NR & - & $\begin{array}{l}\text { Evalúan evolución en el tiempo de los } \\
\text { biomarcadores incluido mioglobina }\end{array}$ \\
\hline $\begin{array}{l}\text { Kennedy et al., } \\
1997^{\text {b } 19}\end{array}$ & $\begin{array}{l}\text { prueba: } 77.3 \% \\
\text { valid: } 52.4 \%\end{array}$ & $\begin{array}{l}\text { prueba: } 86.6 \% \\
\text { valid: } 80 \%\end{array}$ & $\begin{array}{l}\text { prueba: } \\
84.3 \% \\
\text { valid: } \\
73.6 \%\end{array}$ & - & $\begin{array}{l}\text { Analiza una población de difícil } \\
\text { diagnóstico, con ECG sin cambios de } \\
\text { infarto en } 2 \text { hospitales. Comparado con } \\
\text { análisis discriminante }\end{array}$ \\
\hline $\begin{array}{l}\text { Hedén et al., } \\
1997^{\mathrm{a}} 20\end{array}$ & 86.3 & 65.9 & NR & - & $\begin{array}{l}\text { Para la misma sensibilidad los } \\
\text { cardiólogos tuvieron una especificidad } \\
\text { del } 55.4 \%\end{array}$ \\
\hline $\begin{array}{l}\text { Tsien et al., } \\
\qquad 1998^{\mathrm{a}} 21\end{array}$ & 81.4 & 92.1 & 89.9 & VPP: 72.9 & $\begin{array}{l}\text { Contó con datos de } 2 \text { hospitales, se } \\
\text { comparó con el algoritmo computacional } \\
\text { de Goldman y un trabajo previo, así } \\
\text { como con } 2 \text { modelos de RL }\end{array}$ \\
\hline Aase, 1999a 22 & 84.3 & 77.3 & 79.6 & - & $\begin{array}{l}\text { Fue usado en urgencias como un sistema } \\
\text { de soporte a las decisiones clínicas }\end{array}$ \\
\hline $\begin{array}{l}\text { Dreiseitl et al., } \\
\quad 1999^{\mathrm{a}} 23\end{array}$ & NR & NR & NR & $\begin{array}{l}\text { AUC: RN } \\
0.95, \text { RB } \\
0.96, \text { RS } \\
0.95\end{array}$ & $\begin{array}{l}\text { Compararon contra una regresión } \\
\text { logística }\end{array}$ \\
\hline $\begin{array}{l}\text { Lu et al., } 2000^{\mathrm{b}} \\
\end{array}$ & 84.6 & 90 & - & - & $\begin{array}{l}\text { En la publicación no se hace la } \\
\text { descripción de la población }\end{array}$ \\
\hline $\begin{array}{l}\text { Wang et al., } \\
\qquad 2001^{\text {b } 25}\end{array}$ & NR & NR & NR & $\begin{array}{l}\text { AUC: } \text { prueba } \\
0.83 \text { valid } \\
0.85\end{array}$ & $\begin{array}{l}\text { Bases de datos de } 2 \text { hospitales tomando } \\
\text { al segundo como validación. } \\
\text { Rendimiento similar al de RL }\end{array}$ \\
\hline $\begin{array}{l}\text { Baxt et al., } \\
\qquad 2002-1^{\text {a }} 26\end{array}$ & 94 & 93.3 & NR & AUC: 0.98 & Se analiza el efecto de la falta de datos \\
\hline $\begin{array}{l}\text { Baxt et al., } \\
\qquad 2002-2 \text { a } 27\end{array}$ & 88.1 & 86.2 & NR & AUC: 0.90 & $\begin{array}{l}\text { Comparado contra el } \mathrm{ACl} \text {-TIPI, algoritmo } \\
\text { de Goldman y una regresión logística }\end{array}$ \\
\hline $\begin{array}{l}\text { Olsson et al., } \\
2002^{\mathrm{a} 28}\end{array}$ & 87 & 45 & - & - & $\begin{array}{l}\text { Casos de pacientes con bloqueo } \\
\text { completo de rama izquierda en el ECG }\end{array}$ \\
\hline
\end{tabular}


Tabla 2 (continuación)

\begin{tabular}{|c|c|c|c|c|c|}
\hline Estudio & Sensibilidad & Especificidad & Precisión & Otro reporte & Comentarios \\
\hline $\begin{array}{l}\text { Haraldsson } \\
\text { et al., 2004 } 29\end{array}$ & NR & NR & NR & AUC: 0.843 & $\begin{array}{l}\text { Comparan varias estrategias de análisis } \\
\text { de adquisición de la información de los } \\
\text { ECG, sin ser superior ninguna }\end{array}$ \\
\hline $\begin{array}{l}\text { Harrison y } \\
\text { Kennedy, } \\
2004^{\text {a } 30}\end{array}$ & $\begin{array}{l}\text { Prueba: } 93 \% \text {, } \\
\text { valid: } 90 \%\end{array}$ & $\begin{array}{l}\text { Prueba: } 93 \% \text {, } \\
\text { valid: } 89 \%\end{array}$ & NR & $\begin{array}{l}\text { AUC: prueba } \\
0.93 \text {, valid } \\
0.95\end{array}$ & $\begin{array}{l}\text { Se comparó contra una RL que presentó } \\
\text { un rendimiento similar }\end{array}$ \\
\hline $\begin{array}{l}\text { Conforti et al., } \\
2005^{\text {b } 31}\end{array}$ & NR & NR & 96.2 & - & $\begin{array}{l}\text { No describe cómo se hizo el diagnóstico, } \\
\text { el kernel polinomial tuvo el mejor } \\
\text { desempeño }\end{array}$ \\
\hline $\begin{array}{l}\text { Bulgiba et al., } \\
2006^{\text {a } 32}\end{array}$ & NR & NR & NR & AUC: 0.792 & $\begin{array}{l}\text { Comparan las redes entrenadas por los } \\
\text { datos extraídos por PCA vs. la } \\
\text { recomendación del experto }\end{array}$ \\
\hline $\begin{array}{l}\text { Coskun et al., } \\
2006^{\text {a } 33}\end{array}$ & 100 & 60 & 81 & - & $\begin{array}{l}\text { Lo comparan contra los médicos de } \\
\text { urgencias que logran una precisión del } \\
85 \%\end{array}$ \\
\hline $\begin{array}{l}\text { Olsson et al., } \\
\qquad 2006^{\text {a } 34}\end{array}$ & 95 & 88 & - & AUC: 0.98 & $\begin{array}{l}\text { Mejoraba el rendimiento diagnóstico de } \\
\text { los médicos al tener en cuenta su } \\
\text { reporte. Concordancia con cardiólogos } \kappa \\
\text { de } 0.71 \text {. }\end{array}$ \\
\hline $\begin{array}{l}\text { Green et al., } \\
2006^{\text {a } 35}\end{array}$ & NR & NR & NR & $\begin{array}{l}\text { AUC: } \\
\text { Bagging } \\
81.1 \% \text {, } \\
\text { Cross- } \\
\text { spliting } \\
81 \% \text {. }\end{array}$ & $\begin{array}{l}\text { El mejor rendimiento se obtuvo cuando } \\
\text { los datos de entrada fueron solo del } \\
\text { ECG, los } 2 \text { sistemas de ensamble fueron } \\
\text { casi equivalentes. Bajo desempeño de la } \\
\text { RL. El AUC de una ANN fue del } 80 \% \text { y de } \\
\text { RL del } 76.4 \%\end{array}$ \\
\hline $\begin{array}{l}\text { Conforti et al., } \\
2007^{\text {a }} 36\end{array}$ & NR & NR & 93.8 & - & $\begin{array}{l}\text { En este caso el mejor kernel en pruebas } \\
\text { fue el laplasiano. El C } 4.5 \text { tuvo un } 93 \% \text {, } \\
\text { Bayes un } 91.7 \% \text { y RN un } 90.1 \%\end{array}$ \\
\hline $\begin{array}{l}\text { Al-Naima et al., } \\
\qquad 2008^{\text {b } 37}\end{array}$ & 90 & NR & 90 & - & $\begin{array}{l}\text { Multicéntrico, } 2 \text { hospitales. Solo basa su } \\
\text { diagnóstico en la derivación DIII. }\end{array}$ \\
\hline $\begin{array}{l}\text { Forberg et al., } \\
\qquad 2009^{a} 38\end{array}$ & 95 & 44 & NR & AUC: 0.86 & $\begin{array}{l}\text { Le fue mejor a la regresión logística } \\
\text { (AUC } 0.88 \text { y especificidad del } 54 \% \text { ), a los } \\
\text { expertos el AUC fue de } 0.78 \text { sensibilidad } \\
\text { del } 82 \% \text { y especificidad del } 63 \%\end{array}$ \\
\hline $\begin{array}{l}\text { Ainon et al., } \\
2012^{\text {a } 39}\end{array}$ & NR & NR & NR & AUC: 0.75 & $\begin{array}{l}\text { Extraen los datos de los registros } \\
\text { electrónicos de la historia clínica. } \\
\text { Comparan con RN con AUC } 0.79\end{array}$ \\
\hline $\begin{array}{l}\text { Chang et al., } \\
2012^{\text {b } 40}\end{array}$ & NR & NR & 83 & - & $\begin{array}{l}\text { Pobre referencia de la base de datos, la } \\
\text { precisión de la SVM fue del } 71 \%\end{array}$ \\
\hline $\begin{array}{l}\text { Dhawan et al., } \\
\qquad 2012^{\text {b } 41}\end{array}$ & 86.8 & 95 & NR & - & $\begin{array}{l}\text { Comparado con cardiólogos que tuvieron } \\
\text { una sensibilidad del } 55.3 \% \text { y } \\
\text { especificidad del } 83.7 \%\end{array}$ \\
\hline $\begin{array}{l}\text { Salari et al., } \\
\qquad 2013^{\text {a }} 42\end{array}$ & NR & NR & 83.2 & - & $\begin{array}{l}\text { El mejor resultado lo tuvo la RN, seguido } \\
\text { por } 7 \mathrm{NN} \text {. El Bagging de ID3 tuvo mejor } \\
\text { resultado que el ID3 solo }\end{array}$ \\
\hline $\begin{array}{l}\text { Vila-Francés } \\
\text { et al., } 2013^{\text {a } 43}\end{array}$ & 87.1 & 61.1 & 68.9 & $\begin{array}{l}\text { AUC: Bayes } \\
0.72 \text {, FAN } \\
0.74\end{array}$ & $\begin{array}{l}\text { El mejor resultado lo tuvieron las redes } \\
\text { bayesianas aunque no reportan su AUC }\end{array}$ \\
\hline
\end{tabular}

ANN: redes neuronales artificiales; AUC: área bajo la curva ROC; CPK: creatinfosfocinasa; ECG: electrocardiograma; FAN: Forest Augmented Network; LDH: lactato deshidrogenasa; PCA: análisis de componentes principales; RL: regresión logística; RN: red neuronal; SVM: máquina de soporte de vectores.

a Revistas médicas.

b Revistas de ingeniería. 


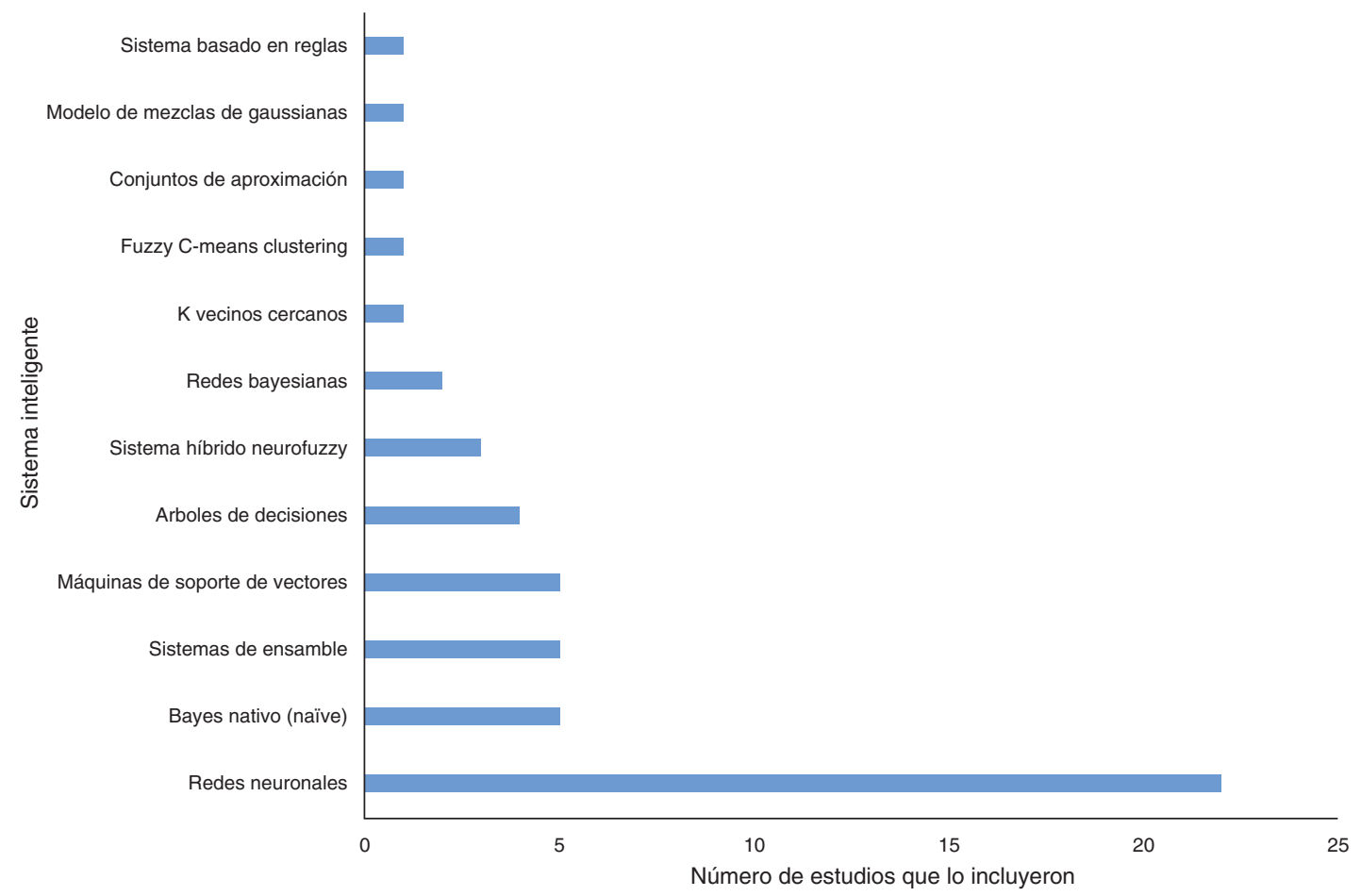

Figura 3 Distribución de la frecuencia de las herramientas de sistemas inteligentes usadas.

con regresión logística $21,23,25,27,30,35,38$. En 4 casos se comparó con el desempeño de los médicos de urgencias $^{9,33,34,38}$, en 3 casos con cardiólogos ${ }^{17,18,41}$ y en 2 casos se compararon con algoritmos computacionales (Goldman y ACl-TIPI) ${ }^{21,27}$.

En la mayor parte de los casos se alcanzaron altos niveles de precisión para el diagnóstico (tabla 2), encontrando el mejor rendimiento en el caso de varias redes neuronales con precisiones tan altas como el 96 y $98 \%^{9,13,26,31}$.

\section{Discusión}

El primer intento documentado para desarrollar y aplicar herramientas computacionales para el soporte del diagnóstico de los SCA fue quizá el expuesto por el grupo de Pozen en $1980^{44,45}$. Aún hoy, luego de 35 años de intensa búsqueda de herramientas de apoyo al diagnóstico de la entidad que da cuenta de la mayor mortalidad en el mundo, no se encuentra una solución definitiva a este tema. Los sistemas inteligentes o de reconocimiento estadístico de patrones se constituyen en una ruta prometedora. Los resultados expuestos demuestran que, por lo general, son mejores que los expuestos para las reglas de predicción clínica que se apoyan en computadores ${ }^{4}$ o son independientes de ellos ${ }^{46}$.

En la revisión realizada se encontraron 35 artículos en los que se evaluaron herramientas de sistemas inteligentes en el diagnóstico de los SCA. A través de la experiencia previa, este número no logra abarcar por completo los trabajos que abordan este tema. Debe decirse al respecto que las bases de datos procedentes de ingeniería no tienen estandarizados los términos usados como palabras clave, de manera opuesta a lo que pasa en las que proceden de medicina. Esta situación parece ser más notoria en los artículos presentados en congresos o conferencias. Como es de esperarse, los artículos que proceden de medicina hacen una descripción más completa de la población que es motivo de estudio y del referente para diagnóstico, mientras que en los de ingeniería tiende a descuidarse estos puntos y darse prelación a la descripción detallada de los aspectos metodológicos y de desarrollo del sistema.

Por tratarse de herramientas desarrolladas de manera automática a partir de la base de datos con la que se alimenta, en la que los datos de ingreso están pareados con el diagnóstico, el aspecto de flujo y tiempo por lo general no constituía riesgo de sesgo a pesar de conocerse el diagnóstico del referente de antemano. Se encuentra una amplia variabilidad en sus resultados con un mejor rendimiento aparente en el caso de ANN y SVM en comparación con las herramientas estadísticas de reconocimiento de patrones y árboles de decisiones (tabla 2). Cuando se comparó con los médicos, su resultado siempre fue superior a ellos. En los estudios en los que se podía establecer de antemano un umbral (principalmente en ANN) se dio prelación a una sensibilidad alta sacrificando la especificidad con el fin de favorecer el diagnóstico de los casos positivos.

Desde el planteamiento de la revisión se esperaba una amplia heterogeneidad entre los estudios, por lo que desde el principio se descartó la realización de un metaanálisis. Las poblaciones investigadas eran diversas, los criterios aplicados como referentes variaron con el paso del tiempo, se consideraban en algunos los biomarcadores o ECG, mientras en otros solo correspondía a datos clínicos y, aun usando la misma herramienta, el método de entrenamiento y arquitectura tiene un gran nivel de variación.

Al analizar la escasa penetración de estos sistemas en la práctica médica Richardson ${ }^{46}$ propone 3 categorías de barreras para el diagnóstico clínico basado en la evidencia: desde el punto de vista de la evidencia disponible, aspectos del 
Tabla 3 Valoración del riesgo de sesgos mediante la herramienta QUADAS 2

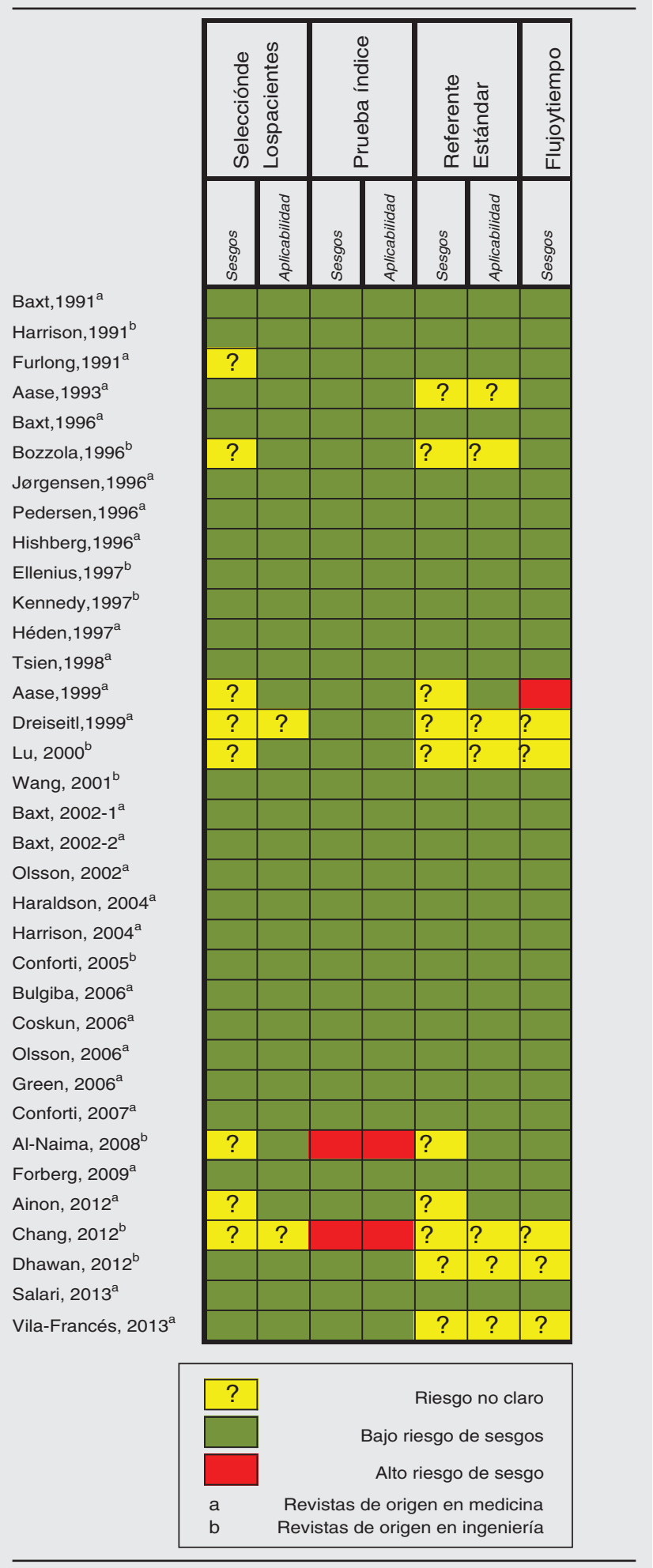

diagnóstico y del sistema de salud.Al considerar el primero de estos puntos, la investigación realizada nos aporta una visión general acerca de la evidencia disponible, es claro que existe una buena cantidad de estudios que vienen llevándose a cabo desde hace bastante tiempo, pero que por lo general no son fácilmente disponibles para el profesional de la salud que finalmente tendrá a su cargo la toma de dichas decisiones. Parece ser visto como un experimento aislado, desligado de la práctica.

Una buena parte de los estudios que se encuentran en bases de datos procedentes de la ingeniería presentan limitaciones para el acceso y su consulta por parte del personal de la salud, y a no ser que sea la línea de trabajo habitual de este, no es tenida en cuenta. Vemos además que el enfoque de las publicaciones es amplio, consideran diversos espectros de la enfermedad, que van desde la isquemia inducida en laboratorio ${ }^{41}$, telemedicina ${ }^{33}$, triage inicial ${ }^{32}$ hasta la utilidad como punto definitorio de la conducta posterior ${ }^{22,34}$. En la mayor parte de ellos no se contemplan otras preguntas diagnósticas como los diagnósticos diferenciales.

Un aspecto fundamental que debe tenerse en cuenta es que existe la posibilidad que los estudios de precisión diagnóstica evalúen solo una parte del desempeño de las herramientas de soporte a las decisiones en el diagnóstico. En lugar de buscar niveles casi perfectos de sensibilidad y especificidad, otro camino podrían ser los estudios en los que se ponga de manifiesto la mejoría en la tasa de aciertos al momento de asignar a los pacientes a cierta área de hospitalización o decidir su alta. Como ejemplo presentamos los trabajos de Jonsbu et al. ${ }^{47}$ que expone como su herramienta mejora en la práctica la asignación de los pacientes en comparación con la forma tradicional, y el de Wilson et al. ${ }^{48}$ que incluye el dato de la lectura automática del ECG en la decisión de trombólisis prehospitalaria en pacientes con infarto. Ambos estudios fueron excluidos al no mostrar datos de precisión diagnóstica.

La interpretación de los hallazgos de los ECG presenta sus problemas particulares; es así como en una revisión sistemática se concluyó que tanto los médicos de diferentes especialidades como el software cometen frecuentemente errores en dicha interpretación ${ }^{49}$. En casi un tercio de los estudios de la revisión, solo se consideró como datos de entrada al sistema la señal electrocardiográfica, sometida o no a algún sistema de procesamiento; sus resultados muestran un desempeño muy bueno para el caso específico de los SCA.

Al considerar las limitaciones de la revisión encontramos que no hay una estandarización de los términos al interior de las bases de datos con lo cual es probable que algunos artículos hayan quedado fuera. A través del tiempo se ha dado una evolución en las definiciones de los SCA lo que hace que el referente no pueda ser aplicable a todos los trabajos de una forma uniforme. Por otro lado, se encuentra una heterogeneidad en la manera como es descrito el rendimiento diagnóstico de las pruebas evaluadas y en la mayor parte de los casos no se puede elaborar tablas de contingencia para ilustrar los falsos y verdaderos positivos/negativos.

Los sistemas inteligentes ya hacen parte de nuestra vida cotidiana. En medicina, el desarrollo más importante se ha centrado en el reconocimiento automático de imágenes especialmente en radiología, big data enfocado hacia farmacovigilancia y modelamiento de epidemias en salud pública. El presente trabajo expone la el extenso camino llevado a cabo en búsqueda de nuevas herramientas que ayuden al diagnóstico de los SCA, sobre todo cuando quien se enfrenta al paciente es personal médico no especializado como ocurre en muchas ocasiones. Aunque aún no se cuente 
con una aplicación que haga uso de estas herramientas a pie del paciente, es probable que pronto lo veamos en la práctica gracias al avance importante de la tecnología en los últimos años y la investigación creciente en estos temas.

\section{Conclusiones}

Esta revisión expone cómo las herramientas de sistemas inteligentes tienen alto rendimiento en el diagnóstico de los SCA utilizando diferentes fuentes de entradas como lo son datos clínicos, biomarcadores y ECG así como varias de estas herramientas en donde las redes neuronales son las más utilizadas. Teniendo en cuenta lo descrito se debe considerar a futuro el uso de estas herramientas en la práctica clínica habitual.

\section{Responsabilidades éticas}

Protección de personas y animales. Los autores declaran que para esta investigación no se han realizado experimentos en seres humanos ni en animales.

Confidencialidad de los datos. Los autores declaran que en este artículo no aparecen datos de pacientes.

Derecho a la privacidad y consentimiento informado. Los autores declaran que en este artículo no aparecen datos de pacientes.

\section{Financiación}

Ninguna.

\section{Conflicto de intereses}

Los investigadores dejamos constancia que no poseemos ningún clonflicto de intereses.

\section{Anexo. Material adicional}

Se puede consultar material adicional a este artículo en su versión electrónica disponible en doi:10.1016/j.acmx. 2017.03.002.

\section{Bibliografía}

1. Hamm CW, Bassand JP, Agewall S, et al. ESC Guidelines for the management of acute coronary syndromes in patients presenting without persistent ST-segment elevation: The Task Force for the management of acute coronary syndromes (ACS) in patients presenting without persistent ST-segment elevation of the European Society of Cardiology (ESC). Eur Heart J. 2011;32:2999-3054.

2. Lobach D, Sanders GD, Bright TJ, et al. Enabling health care decisionmaking through clinical decision support and knowledge management. Evid Rep Technol Assess. 2012:1-784.

3. Negnevitsky M. Artificial intelligence. A guide to intelligent systems. 2. ${ }^{\mathrm{a}}$ ed Harlow: Addison Wesley; 2004. p. 440.

4. Steurer J, Held U, Schmid D, et al. Clinical value of diagnostic instruments for ruling out acute coronary syndrome in patients with chest pain: A systematic review. Emerg Med J. 2010;27:896-902.

5. Lee AJ, Michail M, Quaderi SA, et al. Implementation of NICE Clinical Guideline 95 for assessment of stable chest pain in a rapid access chest pain clinic reduces the mean number of investigations and cost per patient. Open Heart. 2015;2:e000151.

6. Skinner JS, Smeeth L, Kendall JM, et al., Chest Pain Guideline Development Group. NICE guidance. Chest pain of recent onset: Assessment and diagnosis of recent onset chest pain or discomfort of suspected cardiac origin. Heart. 2010;96: 974-8.

7. Amsterdam EA, Kirk JD, Bluemke DA, et al. Testing of low-risk patients presenting to the emergency department with chest pain: A scientific statement from the American Heart Association. Circulation. 2010;122:1756-76.

8. Whiting PF, Rutjes AW, Westwood ME, et al. QUADAS-2: A revised tool for the quality assessment of diagnostic accuracy studies. Ann Intern Med. 2011;155:529-36.

9. Baxt WG. Use of an artificial neural network for the diagnosis of myocardial infarction. Ann Intern Med. 1991;115:843-8.

10. Harrison RF, Marshall SJ, Kennedy RL. The early diagnosis of heart attacks: A neurocomputational approach. Seattle: IEEE; 1991. p. 1-5.

11. Furlong JW, Dupuy ME, Heinsimer JA. Neural network analysis of serial cardiac enzyme data. A clinical application of artificial machine intelligence. Am J Clin Pathol. 1991;96:134-41.

12. Aase $\mathrm{O}$, Jonsbu J, Liestøl K, et al. Decision support by computer analysis of selected case history variables in the emergency room among patients with acute chest pain. Eur Heart J. 1993;14:433-40.

13. Baxt WG, Skora J. Prospective validation of artificial neural network trained to identify acute myocardial infarction. Lancet. 1996;347:12-5.

14. Bozzola P, Bortolan G, Combi C, et al. A hybrid neuro-fuzzy system for ECG classification of myocardial infarction. Comput Cardiol. 1996:241-4.

15. Jørgensen JS, Pedersen JB, Pedersen SM. Use of neural networks to diagnose acute myocardial infarction. I. Methodology. Clin Chem. 1996;42:604-12.

16. Pedersen SM, Jørgensen JS, Pedersen JB. Use of neural networks to diagnose acute myocardial infarction. II. A clinical application. Clin Chem. 1996;42:613-7.

17. Hirshberg AJ, Guttman TG. Criteria-based expert system for cardiac ischemia evaluation in the emergency department. Acad Emerg Med. 1996;3:689-93.

18. Ellenius J, Groth T, Lindahl B, et al. Early assessment of patients with suspected acute myocardial infarction by biochemical monitoring and neural network analysis. Clin Chem. 1997; 43:1919-25.

19. Kennedy RL, Harrison RF, Burton AM, et al. An artificial neural network system for diagnosis of acute myocardial infarction (AMI) in the accident and emergency department: Evaluation and comparison with serum myoglobin measurements. Comput Methods Programs Biomed. 1997;52:93-103.

20. Hedén B, Öhlin H, Rittner R, et al. Acute myocardial infarction detected in the 12-lead ECG by artificial neural networks. Circulation. 1997;96:1798-802.

21. Tsien CL, Fraser HS, Long WJ, et al. Using classification tree and logistic regression methods to diagnose myocardial infarction. Stud Health Technol Inform. 1998;52 Pt 1:493-7.

22. Aase O. Clinical experience with a decision support computer program using Bayes' theorem to diagnose chest pain patients. Cardiology. 1999;92:128-34.

23. Dreiseitl S, Ohno-Machado L, Vinterbo S. Evaluating variable selection methods for diagnosis of myocardial infarction. Proc AMIA Symp. 1999:246-50.

24. Lu HL, Ong K, Chia P. Automated ECG classification system based on a neuro-fuzzy system. Comput Cardiol. 2000;27:387-90. 
25. Wang SJ, Ohno-Machado L, Fraser HS, et al. Using patientreportable clinical history factors to predict myocardial infarction. Comput Biol Med. 2001;31:1-13.

26. Baxt WG, Shofer FS, Sites FD, et al. A neural computational aid to the diagnosis of acute myocardial infarction. Ann Emerg Med. 2002;39:366-73.

27. Baxt WG, Shofer FS, Sites FD, et al. A neural network aid for the early diagnosis of cardiac ischemia in patients presenting to the emergency department with chest pain. Ann Emerg Med. 2002;40:575-83.

28. Olsson SE, Ohlsson M, Ohlin $\mathrm{H}$, et al. Neural networks-a diagnostic tool in acute myocardial infarction with concomitant left bundle branch block. Clin Physiol Funct Imaging. 2002;22:295-9.

29. Haraldsson H, Edenbrandt L, Ohlsson M. Detecting acute myocardial infarction in the 12-lead ECG using Hermite expansions and neural networks. Artif Intell Med. 2004;32:127-36.

30. Harrison RF, Kennedy RL. Artificial neural network models for prediction of acute coronary syndromes using clinical data from the time of presentation. Ann Emerg Med. 2005;46: 431-9.

31. Conforti D, Guido R. Kernel-based support vector machine classifiers for early detection of myocardial infarction. Optim Methods Softw. 2005;20:401-13.

32. Bulgiba AM. Using neural networks and just nine patientreportable factors of screen for AMI. Health Informatics J. 2006;12:213-25.

33. Coskun O, Eren A, Eren M. A computer based telemedicine protocol to predict acute coronary syndrome in patients with chest pain at home. Int Heart J. 2006;47:491-500.

34. Olsson SE, Ohlsson M, Ohlin H, et al. Decision support for the initial triage of patients with acute coronary syndromes. Clin Physiol Funct Imaging. 2006;26:151-6.

35. Green M, Björk J, Forberg J, et al. Comparison between neural networks and multiple logistic regression to predict acute coronary syndrome in the emergency room. Artif Intell Med. 2006;38:305-18.

36. Al-Naima FM, Ali AH, Mahdi SS. Data acquisition for myocardial infarction classification based on wavelets and Neural Networks. In IEEE SSD 2008. 5th International Multi-Conference on Systems, Signals and Devices; 2008. p. 1-6.
37. Al-Naima FM, Ali AH, Mahdi SS. Data acquisition for myocardial infarction classification based on wavelets and Neural Networks. Seattle: IEEE; 2008. p. 1-6.

38. Forberg JL, Green M, Björk J, et al. In search of the best method to predict acute coronary syndrome using only the electrocardiogram from the emergency department. J Electrocardiol. 2009;42:58-63.

39. Ainon RN, Bulgiba AM, Lahsasna A. AMI screening using linguistic fuzzy rules. J Med Systems. 2010;36:463-73.

40. Chang P-C, Lin J-J, Hsieh J-C. Myocardial infarction classification with multi-lead ECG using hidden Markov models and Gaussian mixture models. Appl Soft Comput. 2012;12:3165-75.

41. Dhawan A, Wenzel B, George S, et al. Detection of acute myocardial infarction from serial ECG using multilayer support vector machine. Conf Proc IEEE Eng Med Biol Soc. 2012;2012:2704-7.

42. Salari N, Shohaimi S, Najafi F, et al. Application of pattern recognition tools for classifying acute coronary syndrome: An integrated medical modeling. Theor Biol Med Model. 2013;10:57.

43. Vila-Francés J, Sanchís J, Soria-Olivas E, et al. Expert system for predicting unstable angina based on Bayesian networks. Expert Syst Appl. 2013;40:5004-10.

44. Pozen MW, D'Agostino RB, Mitchell JB, et al. The usefulness of a predictive instrument to reduce inappropriate admissions to the coronary care unit. Ann Intern Med. 1980; 92 (2 Pt 1): 238-42.

45. Hess EP, Thiruganasambandamoorthy V, Wells GA, et al. Diagnostic accuracy of clinical prediction rules to exclude acute coronary syndrome in the emergency department setting: a systematic review. CJEM. 2008; 10: 373-82.

46. Richardson WS. We should overcome the barriers to evidencebased clinical diagnosis! J Clin Epidemiol. 2007;60:217-27.

47. Jonsbu J, Aase O, Rollag A, et al. Prospective evaluation of an EDB-based diagnostic program to be used in patients admitted to hospital with acute chest pain. Eur Heart J. 1993;14:441-6.

48. Wilson RE, Kado HS, Percy RF, et al. An algorithm for identification of ST-elevation myocardial infarction patients by emergency medicine services. Am J Emerg Med. 2013;31:1098-102.

49. Salerno SM, Alguire PC, Waxman HS. Competency in interpretation of 12-lead electrocardiograms: A summary and appraisal of published evidence. Ann Intern Med. 2003;138:751-60. 